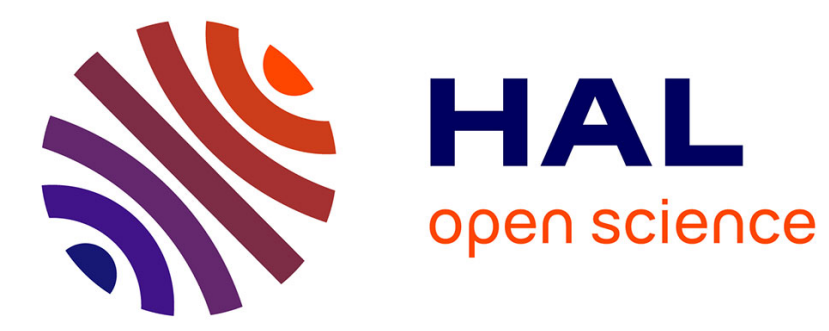

\title{
Some Remarks on Wavelet Representations and Geometric Aspects
}

Bruno Torrésani

\section{To cite this version:}

Bruno Torrésani. Some Remarks on Wavelet Representations and Geometric Aspects . Wavelets: Theory, Algorithms and Applications, Oct 1993, Taormina, Italy. hal-01739594

\section{HAL Id: hal-01739594 \\ https://hal-amu.archives-ouvertes.fr/hal-01739594}

Submitted on 21 Mar 2018

HAL is a multi-disciplinary open access archive for the deposit and dissemination of scientific research documents, whether they are published or not. The documents may come from teaching and research institutions in France or abroad, or from public or private research centers.
L'archive ouverte pluridisciplinaire HAL, est destinée au dépôt et à la diffusion de documents scientifiques de niveau recherche, publiés ou non, émanant des établissements d'enseignement et de recherche français ou étrangers, des laboratoires publics ou privés. 


\title{
SOME REMARKS ON WAVELET REPRESENTATIONS AND GEOMETRIC ASPECTS
}

\author{
Bruno TORRESANI
}

\begin{abstract}
We consider continuous wavelet decompositions, mainly from geometric and algebraic aspects. As examples we describe a scheme for construction of wavelet decompositions of functions on spaces that are homogeneous with respect to some group action. Restriction to discrete group actions we show how multiresolution structures can be directly derived from algebraic arguments. We finally describe approximate multiresolution structures adapted to cases in which no exact pyramidal algorithm can be associated with a given wavelet.
\end{abstract}

\section{$\oint$ Introduction}

The main goal of this paper is to provide a description of (continuous) wavelet analysis and corresponding algorithms, mainly in terms of algebraic and geometric arguments. Today, sereval different aspects of wavelets have been developed, so that the word "wavelet" have now to be understood in a somewhat wide sense. Depending on the application of wavelets one has in mind, one may be more interested in some particular approaches to wavelets; for example if one needs efficient and fast numerical computations, an appropriate algorithmic sructure is required, which is more or less synonymous of multiresolution approach. If on the other hand one is more interested in signal analysis, one may be interested in considering instead redundant representations, and emphasize their symmetry properties.

This paper is devoted to the description of the latter aspect of wavelet decompositions. More precisely, we will essentially focus on the time-frequency (or higher-dimensional generalizations) representation theorems that can be obtained from covariance requirements. We will also show how the generic algorithmic (multiresolution) structure of wavelet analysis can be derived from group-theoretical arguments (as a consequence of some discrete covariance requirements on the wavelet transform), naturally leading to quadrature mirror 
filters. Finally, we will describe the situation that occurs when no discrete filters are available, but the wavelet and scaling function are enough localized in the fourier space to ensure the existence of approximate discrete filters yielding a good approximation of the wavelet transform.

The main sections of this paper have been written in such a way that they can be read independently from each other (except the third section, that contains examples related to the previous one).

\section{$\S$ The group-theoretical picture}

The group theoretical approach to time-frequency analysis was set first by A. Grossmann, J. Morlet and T. Paul in [10]. The main interest of such an approach is that it emphasizes from the very beginning the covariance properties of the representation. Let us consider for instance the case of one-dimensional signal analysis. It is clear that at least for some purposes such as regognition, the representation has to be translation covariant, in other words the representation of a shifted signal must be a shifted copy of the representation of the signal. The main purpose of the group theoretical approach is to say: let's put the covariance requirement at the very beginning, and derive the representation from it. By covariance we shall always mean covariance with respect to the action of a symmetry group (because we shall always need to consider products and inverse of transformations from the group).

\section{Generalities}

So let us start from a measured space $(X, m)$ and a covariance group $G$. We want to construct representations of functions of $L^{2}(X, m)$, covariant with respect to $G$. This implicitely means that $G$ has a well-defined action on $X$, otherwise stated that $X$ is homogeneous with respect to $G: \forall x, x^{\prime} \in X$, there exists $g \in G$ such that $x^{\prime}=g \cdot x$. We will also require that $m$ is quasi-invariant with respect to the action of $G$, i.e. that the transformed measure $d m(g \cdot x)$ is absolutely continuous with respect to $d m(x)$ (the existence of such quasiinvariant measures is a classical result of harmonic analysis on homogeneous spaces).

Then there is a canonical unitary representation of $G$ on $L^{2}(X, m)$, given by:

$$
\pi(g) \cdot f(x)=\sqrt{\frac{d m\left(g^{-1} \cdot x\right)}{d m(x)}} f\left(g^{-1} \cdot x\right)
$$

Given such a set of data, and a "mother wavelet" $\psi \in L^{2}(X, m)$, there is a natural candidate for wavelet transform, namely the map

$$
\begin{gathered}
T: f \in L^{2}(X, m) \rightarrow T_{f} \in L^{2}(G, \mu) \\
T_{f}(g)=\langle f, \pi(g) \cdot \psi\rangle
\end{gathered}
$$


that "tests" the signal $f(x)$ with all the elementary wavelets

$$
\psi_{(g)}(x)=\sqrt{\frac{d m\left(g^{-1} \cdot x\right)}{d m(x)}} \psi\left(g^{-1} \cdot x\right)
$$

The wavelet transform is a bounded function on $G$ (as follows from the CauchySchwarz inequality); moreover, since the representation is unitary, one also gets "for free" the covariance of the wavelet transform

$$
T_{\pi(h) \cdot f}(g)=T_{f}\left(h^{-1} g\right)
$$

Now arises the question of "invertibility" of the wavelet transform constructed in that way. For this it is convenient to introduce the following operator

$$
\begin{gathered}
\mathcal{A}: f \in L^{2}(X, m) \rightarrow \mathcal{A} \cdot f \\
\mathcal{A} \cdot f(x)=\int_{G} T_{f}(g) \psi_{(g)}(x) d \mu(g)
\end{gathered}
$$

that "inverts" the wavelet transform. Here, $\mu$ is the (unique, up to a constant factor) left-invariant mrasure on $G$ (i.e. $d \mu(h g)=d \mu(g))$. This naturally leads to the following set of questions:

1 When is the group "sufficiently large" to characterize any $f \in L^{2}(X, m)$ ? In other words, when is the $\mathcal{A}$ operator invertible?

2 When is the symmetry group "not too large" ? Otherwise stated, when is the $\mathcal{A}$ operator bounded ?

3 When is $\mathcal{A}$ a multiple of the identity (as happens for instance in the wavelet case), i.e. $T$ is a multiple of an isometry?

4 What does $G$ have to do with "time-frequency" ?

\section{Square-integrability}

The answer to question 3 has been well-known for quite a long time, and deals with the theory of square-integrable representations.

Definition 1 The representation $\pi$ of $G$ is square-integrable if

$1 \pi$ is irreducible.

2 There exists a function $\psi \in L^{2}(X, m)$ such that the set of diagonal matrix elements $\varphi_{\psi}(g)=\langle\psi, \pi(g) \cdot \psi\rangle$ belongs to $L^{2}(G, \mu) . \psi$ is said to be admissible.

The main result for our purpose lies in the following orthonormality relations, proved by Duflo and Moore [6], Carey [4] and Grossmann, Morlet and Paul [10]. Here is a weak form of the result:

Theorem 2 Let $\pi$ be a square-integrable unitary representation of the locally compact group $G$ in $L^{2}(X, m)$, and let $\psi$ be an admissible vector. Then the map

$$
T: f \in L^{2}(X, m) \rightarrow \frac{1}{\sqrt{c_{\psi}}} T_{f} \in L^{2}(G, \mu)
$$


is an isometry, and can then be inverted by its adjoint.

In other words, any function $f \in L^{2}(X, m)$ can be decomposed as a superposition of modified copies of $\psi$, obtained by acting on it with the symmetry group $G$.

Remark. The result of Duflo and Moore is actually much stronger. They in particular show that the set of admissible vectors coincide with the domain of some densely defined linear operator $K$; in addition, $K$ turns out to be scalar whenever $G$ is unimodular.

As an example, one may consider the natural action of the rotation group $S O(3)$ on the 2-dimensional sphere $S^{2}$. Such a representation is not squareintegrable, because it is highly reducible: it splits into a countable infinity of invariant finite-dimensional representation spaces; such a decomposition leads to the well-known spherical harmonics, and is not suitable for our purpose since in addition, it does not yield any natural notion of (local) frequency. However, the notion of frequency can be introduced into the game by considering an extension of the rotation group by local frequency translations, leading to windowed Fourier transform on spheres. We shall come back in more details to this example in the next section, but for our current purpose, it is sufficient to say that such a group (the so-called Euclidian group) is "too large" for the representation space, in other words that the reresentation is not squareintegrable.

\section{Going to quotient spaces}

The case of spheres is particularly interesting because it allows one to really see where the departure from square-integrability comes from. Roughly speaking, it may be said that at any point, the corresponding "local Fourier space" can be identified with the (co)tangent space at this point, so that the space of all (local) frequency translations is isomorphic to $\mathbb{R}^{n}$. Then if one believes that the wavelet representations have something to do with "position-frequency" representations, one has to factor out the extra-dimension to go back to $\mathbb{I}^{n-1}$ (see e.g. [1]).

From a more general point of view, this means that one has to consider systems of vectors in the representation space, labeled by elements of some quotient space $P=G / H$ rather than the group itself. But since the representation $\pi$ is defined on $G$ and not on $P$ (let us recall that $P$ may be viewed as a set of equivalence classes of elements of $G$, two elements $g$ and $g^{\prime}$ being equivalent if $g^{\prime}=g h$ for some $h \in H$ ), one has to choose a representative element $\sigma(p) \in G$ for any $p \in P$. Then one can introduce the generalized coherent states (or wavelets): if $\psi \in L^{2}(X)$

$$
\psi_{(p)}=\pi \circ \sigma(p) \cdot \psi
$$

and again the operator

$$
\mathcal{A}_{\sigma}: f \in L^{2}(X) \rightarrow \int_{P}\left\langle f, \psi_{(p)}\right\rangle \psi_{(p)} d \eta(p)
$$


where $\eta$ is the unique (up to a constant factor) quasi-invariant measure on $P$, and again ask the same questions as before, regarding the "size" of $P$ and the properties of $\sigma$.

\section{Phase-Space}

Among the possible candidates for the $P$ space, there is a most natural one, obtained as follows. Let us recall that we started from a representation $\pi$ of $G$. Differentiating $\pi$ (i.e. looking at the representatives of elements close to the identity of $G$ ), one obtains a representation $d \pi$ of the Lie algebra $\mathcal{G}$ of $G$ (i.e. the tangent space at the identity). It turns out that for a large class of groups, the representation $d \pi$ can be constructed from a linear form on $\mathcal{G}$, i.e. an element $F_{\pi} \in \mathcal{G}^{*}$, the dual space of $\mathcal{G}$.

Now, to this $F_{\pi}$ element can be associated a family of vectors $F_{\pi}^{h} \in \mathcal{G}^{*}$ :

$$
F_{\pi}^{h}(X)=F_{\pi}\left(h X h^{-1}\right) \quad \forall X \in \mathcal{G}
$$

By definition, the coadjoint orbit $\mathcal{O}_{\pi}$ associated with the representation $\pi$ is the set of such $F^{h}$ vectors, in other words the quotient of $G$ by the subgroup of elements that leave $F_{\pi}$ invariant.

$$
\mathcal{O}_{\pi} \cong G / G_{\pi}
$$

where

$$
G_{\pi}=\left\{h \in G, F^{h}=F\right\}
$$

It can be shown (see for example [12] for a review) that $\mathcal{O}_{\pi}$ inherits a structure of symplectic manifold. $\mathcal{O}_{\pi}$ is sometimes called the phase space associated with $\pi$ (in the geometrical sense), and is for us a natural candidate to study square-integrability of the representations.

Remark. It must be recalled here that the Lie groups and algebras under consideration are always finite-dimensional. Then the computations with the dual space are easy to do, since the dual can be identified with the space itself. This allows to compute explicitely the phase space associated with a given representation, provided that the representation can be generated from an element $F_{\pi} \in \mathcal{G}^{*}$. Moreover, the groups under consideration can in general be realized as simple matrices groups, so that explicit computations are much easier. For instance, the affine or " $a x+b$ " group in dimension 1 can be realized as the group of matrices of the form

$$
G_{a f f}=\left\{\left(\begin{array}{ll}
a & b \\
0 & 1
\end{array}\right), b \in \mathbb{R}, a \in \mathbb{R}_{+}^{*}\right\}
$$

while the Weyl-Heisenberg group is the group of matrices

$$
G_{W H}=\left\{\left(\begin{array}{ccc}
1 & q & t \\
0 & 1 & p \\
0 & 0 & 1
\end{array}\right), p, q \in \mathbb{R}, t \in[0,2 \pi]\right\}
$$

From such matrix realizations it is easy to compute the matrix realizations of the corresponding Lie algebras (just by differentiating at the origin $C^{1}$ curves of the form $M_{x}, x \in \mathbb{R}$, with $\left.M_{0}=e\right)$. 


\section{$\S$ Examples}

\section{Square-integrable representations}

The first examples are well-known, and yield the usual wavelet transforms. In such cases, the covariance group turns actually out to be isomorphic (up to a compact factor) to the geometric phase space of the representation.

This is the case of the so-called affine (or " $a x+b$ group"), generated by translations and dilations. In such a case, the group law (see the matrix realization above) is the following

$$
(b, a) \cdot\left(b^{\prime}, a^{\prime}\right)=\left(b+a b^{\prime}, a a^{\prime}\right)
$$

and it is well-known that the following representation, acting on the Hardy space $H^{2}(I R)=\left\{f \in L^{2}(I R), \hat{f}(\xi)=0 \forall \xi \leq 0\right\}$

$$
\pi(b, a) \cdot f(x)=\frac{1}{\sqrt{a}} f\left(\frac{x-b}{a}\right):=f_{(b, a)}(x)
$$

is square-integrable. Then the direct application of Duflo-Moore's theorem yields the celebrated Calderón identity. If $\psi \in L^{2}(I R)$ is admissible (i.e. such that $\left.\int_{0}^{\infty}|\hat{\psi}(\xi)|^{2} d \xi / \xi=1\right)$, then any $f \in H^{2}(I R)$ can be decomposed as

$$
f=\int_{0}^{\infty} \int_{\mathbb{R}}\left\langle f, \psi_{(b, a)}\right\rangle \psi_{(b, a)} \frac{d a}{a} \frac{d b}{a}
$$

The extension to the $L^{2}(I R)$ context is trivially obtained under the additional assumption $\int_{0}^{\infty}|\hat{\psi}(-\xi)|^{2} d \xi / \xi=1$.

The set of admissible vectors is moreover isomorphic to the domain of a linear operator $K$, which is in this case a convolution operator, defined by the symbol $\sigma(\xi)=\xi^{-1 / 2}$.

The generalizations of affine wavelets to higher dimensions have also been considered by Murenzi in a specific case [15], and later on by Bernier and Taylor [3]. The key point is that to keep an irreducible representation, the scale parameter has to be replaced by matrices, i.e. one has to consider subgroups of $G L(n)$. Let $H$ be such a subgroup, and consider

$$
G=H \times \mathbb{R}^{n}
$$

with group multiplication given by

$$
(x, h) \cdot\left(x^{\prime}, h^{\prime}\right)=\left(x+h \cdot x^{\prime}, h h^{\prime}\right)
$$

$G$ can be realized as a matrix group as follows:

$$
G=\left\{\left(\begin{array}{ll}
h & b \\
0 & 1
\end{array}\right), b \in \mathbb{R}^{n}, a \in H\right\}
$$


where $b$ is understood as a column vector, and 0 as a line vector of length $n$.

Then let $U=H \cdot x_{0}$ be an orbit of $H$ in $\mathbb{R}^{n}$, and let $H_{U}^{2}\left(\mathbb{R}^{2}\right)$ be the subspace of $L^{2}\left(I^{n}\right)$ consisting of functions whose Fourier transform is supported in $U$. This is a possible generalization of the classical complex Hardy space $H^{2}(I R)$ to the multidimensional setting. Then Bernier and Taylor show that if the $U$-orbit is free (i.e. if $h \cdot x_{0}=x_{0}$ implies $h=e$ ) and open in $\mathbb{R}^{n}$, the restriction to $H_{U}^{2}\left(I^{2}\right)$ of the natural representation

$$
\pi(h, b) \cdot f(x)=\delta(h)^{-1 / 2} f\left(h^{-1} \cdot(x-b)\right)
$$

is square-integrable (here $\delta(h)=|\operatorname{det} h|$ is the Radon-Nikodym derivative $d(h$. $x) / d x)$. Notice that the assumptions on $H$ force $H$ to be $n$-dimensional. But it is very likely that the results can be generalized to weaker assumptions. For instance, for groups of the form $\mathbb{R}_{+}^{*} \times S O(n) \times \mathbb{R}^{n}$, the representation given in equation (19) is also square-integrable [15], although $\operatorname{dim}(S O(n))>n$ for $n>2$.

This case is particularly interesting, and has been studied in great details. It leads to rotation-covariant wavelet representations, that were developed by R. Murenzi. More precisely, let us stick to the two-dimensional case. The plane $\mathbb{R}^{2}$ is clearly an open free orbit for $\mathbb{R}_{+}^{*} \times S O(2)$. If one denotes by $r(\theta)$ the rotation matrix of angle $\theta$ in the plane, then

$$
d m(r)=d m(\theta)=d \theta
$$

is the rotation-invariant measure on $S O(2)$. One then has the following representation theorem for functions on the plane: if $\psi \in L^{2}\left(R^{2}\right)$ is such that $\int_{\mathbb{R}^{2}}|\hat{\psi}(\xi)|^{2} d \xi /\|\xi\|^{2}=1$, any $f \in L^{2}\left(\mathbb{R}^{2}\right)$ can be decomposed as

$$
f=\int_{\mathbb{R}^{2} \times \mathbb{R}_{+}^{*} \times[0,2 \pi]}\left\langle f, \psi_{(b, a, \theta)}\right\rangle \psi_{(b, a, \theta)} \frac{d b}{a^{2}} \frac{d a}{a} d \theta
$$

with the obvious notations. It is important to notice that in such a case, the coefficients $T_{f}(b, a, \theta)=\left\langle f, \psi_{b, a, \theta}\right\rangle$ can be viewed as a position-frequency representation of $f(x)$, with $b$ as position variable and $(1 / a, \theta)$ as polar coordinates representation of frequency. This has been successfully used in image analysis (see e.g. [2] and [9]). In particular, it can be shown (using standard aproximation techniques [9]) that if $f(x)$ can be modeled as

$$
f(x)=A(x) \cos \varphi(x)
$$

then $T_{f}(b, a, \theta)$ is locally maximum near the two sheets $a_{r}(b), \theta_{r}(b) \pm \pi$, where $a_{r}(b)^{-1}$ and $\theta_{r}(b)$ are polar coordinates of the vector $\nabla A(b)$, if $a_{r}(x)|\nabla A(x)| \ll$ $|A(x)|$ and $\left|\nabla^{2} \varphi(x)\right| \ll\left|\nabla \varphi(x)^{2} / \varphi(x)\right|$. This is the two-dimensional generalization of the classical localization properties of the wavelet transform of asymptotic signals. 


\section{Phase-space representations}

Now arises the question of the connexion of such a formalism with phase space, i.e. position-frequency space. It turns out that geometry can yield a very natural notion of phase space, as described before.

\section{Time-frequency atoms}

Consider the simple example of Gabor transform. It is well-known that Gabor transform is naturally associated with the so-called Weyl-Heisenberg group (a 3-dimensional group), i.e. the group generated by time and frequency translations (with group multiplication $(p, q, t) \cdot\left(p^{\prime}, q^{\prime}, t^{\prime}\right)=\left(p+p^{\prime}, q+q^{\prime}, t+\right.$ $\left.\left.t^{\prime}+p q^{\prime} \bmod 2 \pi\right)\right)$. See the matrix realization in the previous section. As a matter of fact, the Gabor transform of a function is in general not considered as a function on the group itself, but rather as a function of time and frequency, i.e. as a function on the phase space, considered as a quotient of the group. However, a direct computation of the phase-space (i.e. the coadjoint orbit) shows that it is isomorphic to $\mathbb{R}^{2} \cong\{(p, q)\}$, i.e. the parameter set of the Gabor transform.

This remark can be generalized to the case of higher-dimensional groups. The simplest example is that of more general time-frequency atoms, generated by time-frequency translations and modulations. This amounts to introduce a larger group, generated by the above-mentioned transformations, called the affine (or inhomogeneous) Weyl-Heisenberg group.

$$
G \cong R^{2} \times \mathbb{R}_{+}^{*} \times[0,2 \pi]
$$

with group multiplication

$$
\left.(p, q, a, t) \cdot\left(p^{\prime}, q^{\prime}, a^{\prime}, t^{\prime}\right)=\left(p+p^{\prime} / a, q+a q^{\prime}, a a^{\prime}, t+t^{\prime}+a p q^{\prime} \bmod 2 \pi\right)\right)
$$

It can be realized as the following matrix group:

$$
G_{a W H} \cong\left\{\left(\begin{array}{ccc}
1 & a q & t \\
0 & a & p \\
0 & 0 & 1
\end{array}\right), p, q \in \mathbb{R}, t \in[0,2 \pi]\right\}
$$

The irreducible representations of such a group are well-known, and there is a natural one, acting on $L^{2}(I R)$, given by

$$
\pi(p, q, a, t) \cdot f(x)=\frac{1}{\sqrt{a}} e^{i(t+p(x-q))} f\left(\frac{x-q}{a}\right)
$$

A simple calculation shows that $\pi$ is not square-integrable. This simply comes from the fact that the considered group is "too big" for the representation space. However, it is also easy to compute the phase space associated with such a representation (see [11]), and to show that it is isomorphic to the quotient space

$$
\mathcal{O}_{\pi} \cong G /\left(R_{+}^{*} \times[0,2 \pi]\right) \cong R^{2}
$$


Let then $\sigma: \mathcal{O}_{\pi} \rightarrow G$ be a piecewise differentiable Borel function. It was shown in [11] that one can find $\sigma$ functions such that the associated $\mathcal{A}_{\sigma}$ operator is a scalar. This is however not the case in general, but it is not very difficult to show that under some very weak assumptions, $\mathcal{A}_{\sigma}$ is bounded with bounded inverse, and then suitable for numerical applications.

Let us assume for instance that on is only interested in introducing a relationship between the frequency and the bandwidth of the analyzing functions. This amounts to consider sections $\sigma: \mathcal{O}_{\pi} \rightarrow G$ of the following form:

$$
\sigma(q, p)=(q, p, \beta(p), 0)
$$

Then

Proposition 3 Let $\sigma$ be a piecewise $C^{1}$ function: $\mathcal{O}_{\pi} \rightarrow G$ given as in eq. (25). Then $\mathcal{A}_{\sigma}$ is a convolution operator, and is a multiple of the identity if and only if $\beta$ is of the form

$$
\beta(p)=\frac{1}{\alpha p+\alpha^{\prime}}
$$

for some constants $\alpha, \alpha^{\prime}$ non simultaneously vanishing.

Proof: A direct computation shows that $\mathcal{A}_{\sigma}$ is indeed a convolution, defined by the multiplyer

$$
\gamma(\xi)=\int_{\mathbb{R}}|\hat{\psi}(\beta(p)(\xi-p))|^{2}|\beta(p)| d p
$$

The end of the proposition follows from the explicit computation of the Jacobian of the change of variable $p \rightarrow \beta(p)(\xi-p)$.

\section{The case of the sphere}

Let us now turn to more complex examples. As was discussed in the beginning of this paper, one is interested in building phase-space harmonic analysis on more general homogeneous spaces. Let us describe now a construction of windowed Fourier analysis on the simplest nontrivial homogeneous space, i.e. the sphere $S^{2}$. Classical harmonic analysis on $S^{2}$ is based on the action of the rotation group $S O(3)$ on $S^{2}$, and leads to spherical harmonics. In order to introduce a notion of local frequency into the analysis, one needs to consider the (co)tangent spaces at any point of the sphere, in other words the cotangent bundle $T^{*} S^{2}$ of the sphere (see figure 1 ).

To build windowed Fourier transform, one needs to introduce in addition frequency translations at any point, i.e. translations in all the planes cotangent to the sphere. This then leads to the translations of $\mathbb{I R}^{3}$, i.e. $\mathbb{I R}^{3}$ itself. Moreover, the action of rotations on frequency translations has to be nontrivial, since when one goes from one point of $S^{2}$ to another one (by a rotation), one has to rotate the cotangent spaces accordingly. 


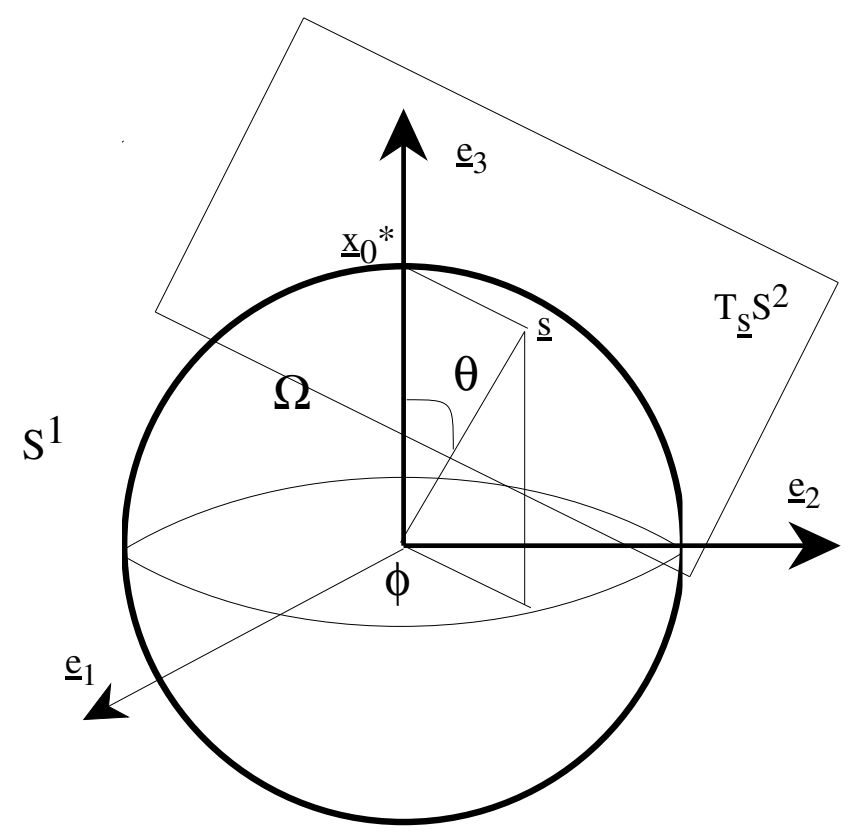

Figure 1: Windowed Fourier analysis on the sphere: geometry of the problem.

Altogether, this leads to the so-called Euclidian group

$$
E(3) \cong S O(3) \times \mathbb{R}^{3}
$$

with the multiplication

$$
(r, p) \cdot\left(r^{\prime}, p^{\prime}\right)=\left(r r^{\prime}, p+r \cdot p^{\prime}\right)
$$

which is a particular case of the groups studied by Bernier and Taylor. It also admits the following matrix realization:

$$
E(3)=\left\{\left(\begin{array}{ll}
r & p \\
0 & 1
\end{array}\right), p \in \mathbb{R}^{n}, r \in S O(3)\right\}
$$

The difference is that one is now no longer interested in its action on $L^{2}\left(R^{3}\right)$ (which leads to Murenzi's wavelets), but on the following representation, acting on $L^{2}\left(S^{2}\right)$ as follows:

$$
\pi(r, p) \cdot f(s)=e^{i p \cdot s} f\left(r^{-1} \cdot s\right) \quad s \in S^{2}
$$

Such a representation is not square-integrable (as can be directly checked). However, as stressed before, there is a natural candidate to restrict the representation to in order to get a position-frequency represntation theorem. Since computations on spheres are somewhat involved, it is simpler to consider the coset space $E(3) / I R$ (which differs from the true phase space, the cotangent bundle of $S^{2}$ only by a compact factor $S O(2)$ that does not change anything to square-integrability). It can be shown that for the considered representation 
Before stating the representation theorem associated with the group action we considered, let us fix our notations. We shall denote by $\Omega$ the upper halfsphere, i.e. the set $\left\{s=(x, y, z) \in \mathbb{R}^{3}, x^{2}+y^{2}+z^{2}=1\right.$ and $\left.z>0\right\}$ and by $\theta$ and $\varphi$ the spherical coordinates of $s \in S^{2}$. Then we have [18]

Theorem 4 Assume that $\psi \in L^{1}\left(S^{2}\right) \cap L^{2}\left(S^{2}\right)$ is such that $\operatorname{Supp}(\psi) \subset \Omega$ and

$$
0 \neq c_{\psi}=8 \pi^{3} \int_{0}^{2 \pi} \int_{-\pi / 2}^{\pi / 2} \frac{|\psi(\theta, \varphi)|^{2}}{\cos \theta} d \theta d \varphi<\infty
$$

Then the map

$$
f \in L^{2}\left(S^{2}\right) \rightarrow \frac{1}{\sqrt{c_{\psi}}} T_{f} \in L^{2}(G / I R)
$$

is an isometry

In other words, any function in $L^{2}\left(S^{2}\right)$ can be decomposed as

$$
f=\int_{\mathbb{R}^{2}} \int_{S O(3)}\left\langle f, \psi_{(r, p)}\right\rangle \psi_{(r, p)} d^{2} p d \mu(r)
$$

i.e. as a superposition of modulated and rotated copies of the window $\psi$. We are then in a situation very close to that of the "flat" windowed Fourier transform; the main modification is that the modulation is now perturbed by the curvature of the sphere.

Remark. The same analysis can also be carried out in any dimension, for instance in the one-dimensional case; the situation is even simpler since the $S O(2)$ rotation group is abelian, and isomorphic to the circle itself. In such a case, one also gets a (periodic) time-frequency representation theorem; the building blocks are of the form

$$
\psi_{(\alpha, p)}(\theta)=e^{i p \sin (\alpha+\theta)} \psi(\alpha+\theta)
$$

where $p \in \mathbb{R}, \alpha, \theta \in[0,2 \pi]$ and $\psi(\theta)$ is a $2 \pi$ periodic function, supported in an interval of length at most $\pi$. The perturbation of the modulation introduced by curvature is even clearer here. Moreover, it is effective only for large values of the angle, since for small $\alpha, \sin \alpha \sim \alpha$. As an example, figure 3 represents the modulus of the windowed Fourier transform on the circle of 125 milliseconds of the speech signal /one/. 


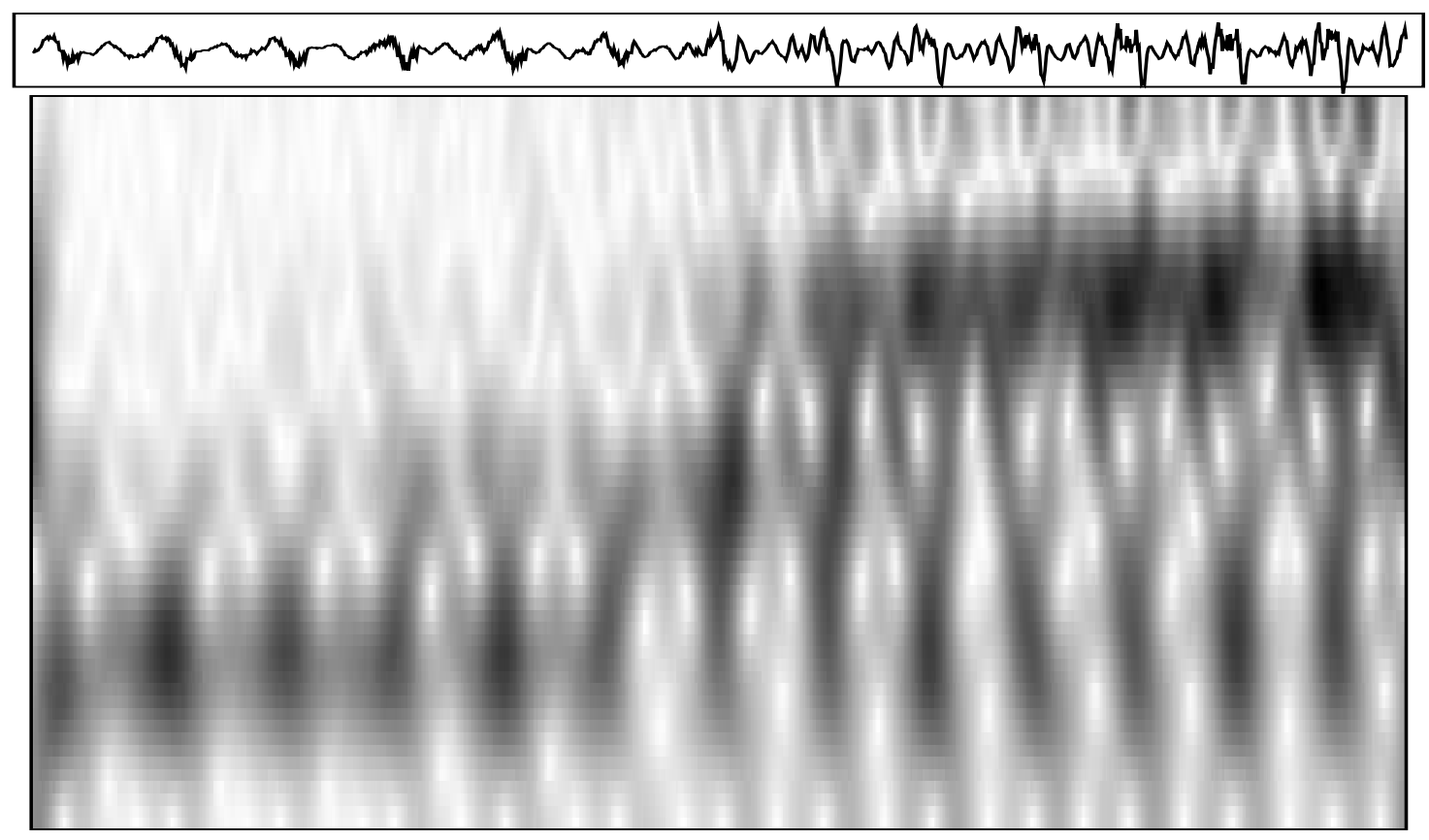

Figure 2: Windowed Fourier transform (modulus) on the circle of $125 \mathrm{~ms}$ of /one/.

Remark. Up to now, it seems that nothing is known about the problem of constructing "true wavelets" on spheres; otherwise stated, how to introduce dilations on spheres.

\section{$\S$ Algorithms from group theoretical considerations}

Let us now go to more algorithmic aspects of wavelets. It is well known that one of the reasons of the success of wavelet analysis is the existence of fast pyramidal algorithms for the computation of the wavelet transform. Such algorithms are of course adapted to discrete problems, and can actually be seen in the following way (this approach is very close to the so-called "algorithme à trous" [8]). Consider a field $\mathbb{I F}=\mathbb{Z}$, or $\mathbb{Z}_{p}$ (the set of congruence classes modulo $p$, where $p$ is a prime number), and the set of sequences on $I F$, that we will denote by $\ell^{2}(I F)$. There exist well-defined translations and dilations on $\ell^{2}(I F)$, according to:

$$
\begin{gathered}
T_{b} \cdot f(n)=f(n-b) \\
D_{a} \cdot f(n)= \begin{cases}f\left(\frac{n}{a}\right) & \text { if } a \text { divides } n \\
0 & \text { elsewhere }\end{cases}
\end{gathered}
$$

Then it can easily be verified that $\left\{T_{b}, D_{a}\right\}$ reproduce the affine multiplication law:

\section{Lemma 5}

$$
T_{b} D_{a} T_{b^{\prime}} D_{a^{\prime}}=T_{b+a b^{\prime}} D_{a a^{\prime}}
$$


Remark. When $F=\mathbb{Z}_{p}$ with $p$ prime, $a$ always divides $n$. Then, there exists a well-defined affine group on $\ell^{2}\left(\mathbb{Z}_{p}\right)$, and the operators $T_{b}$ and $D_{a}$ define a representation of such a group, which turns out to be square-integrable. The general result of Duflo and Moore can then be directly used, and yields a cyclic version of wavelet analysis of sequences, developed by K. Flornes and coworkers in [7].

Moreover, the $D_{a}$-dilation operators are suitable from algorithmic point of view, in the sense that they don't change the number of non-vanishing coefficients. But they have the "drawback of this advantage", in the sense that for example the dilate by 3 of the sequence $\{0, \ldots, 0,1,1,1,0, \ldots 0\}$ is the sequence $\{0, \ldots, 0,1,0,0,1,0,0,1,0, \ldots, 0\}$, which is not convenient if we want to think to this sequence as some sampling of a function defined on the line (or the circle), which is one of the goals of wavelet analysis. An example is shown in figure 2, where one sees the dilates of a sample discrete wavelet. Because of the presence of the intermediate zeroes, it is hard to interpret the discrete dilated wavelets as samplings (whatever it means...) of dilated copies of a function.

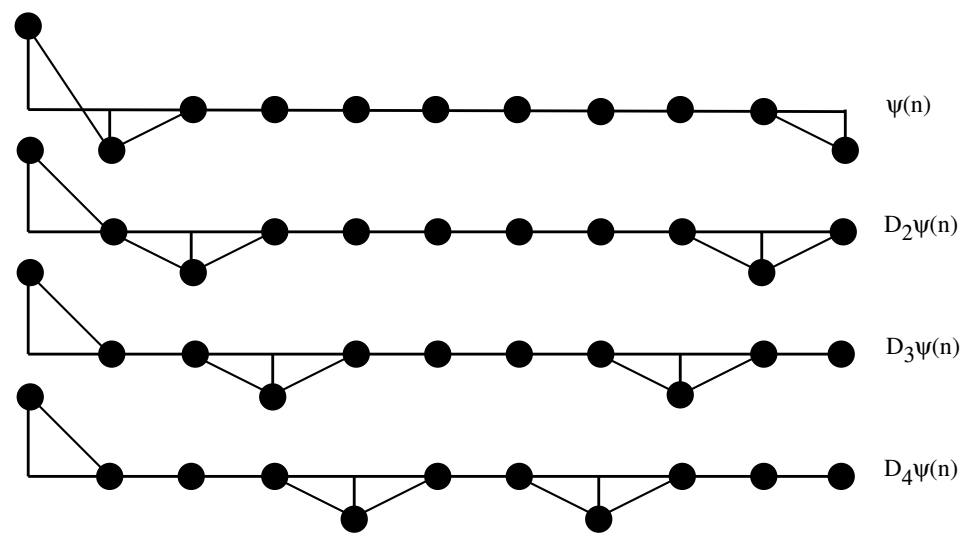

Figure 3:Discrete dilation "with holes".

The effect of such a dilation "with holes" can be seen in figure 4, which represents the cyclic (i.e. on $\mathbb{Z}_{p}$ ) wavelet transform of a "Dirac sequence", i.e. the set of cyclic convolutions products of the periodic sequence $(0, \cdots, 0,1,0, \cdots, 0)$ of length 97 with scaled copies $D_{a} \cdot \psi$ of a real-valued wavelet with 7 nonvanishing coefficients (represented at the bottom of the figure).

To avoid such effects, one needs a dilation operator allowing one:

- To think to sequences as discretization of functions (i.e. maps from $L^{2}(I R)$ to $\ell^{2}(\mathbb{Z})$ for example).

- To think to the discrete dilation as the combination of a continuously defined dilation and the previous discretization.

- To keep the algebraic properties of translations and dilations, i.e. to continue to satisfy equation (37).

Such problem was already addressed in [8], and later on in [7]. 


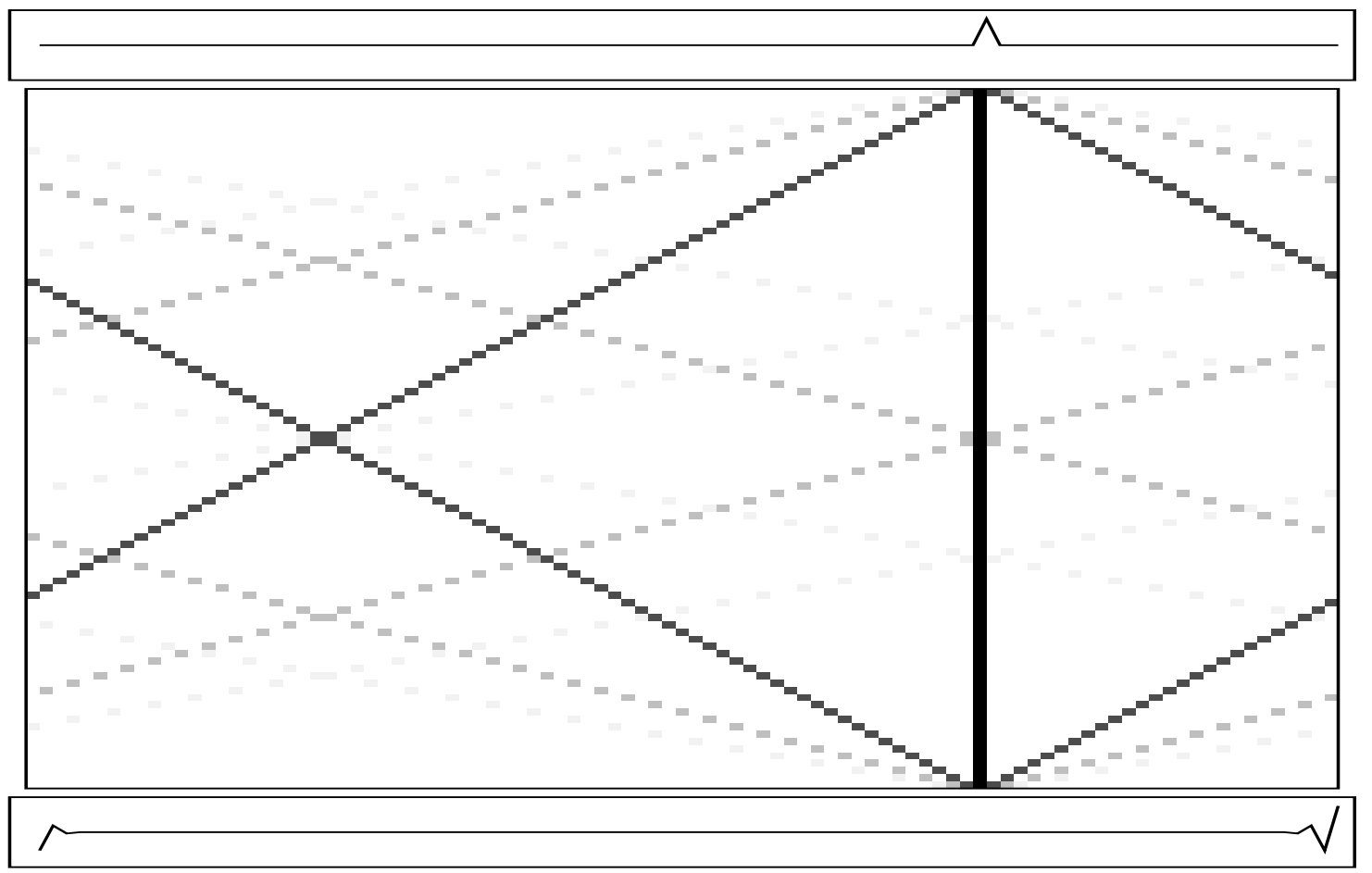

Figure 4: Cyclic wavelet transform of a "Dirac sequence", with the dilation "with holes".

Let us then introduce the following family of "pseudo-dilations"

$$
\mathcal{D}_{a}=K_{a} D_{a}
$$

where $\left\{K_{a}, a \in \mathbb{I F}^{*}\right\}$ is a family of linear operators acting on $\ell^{2}(\mathbb{I F})$.

Definition 6 The family $\left\{\mathcal{D}_{a}, a \in I F^{*}\right\}$ is an admissible family of dilations if the operators $T_{b} \mathcal{D}_{a}$ satisfy the equation (37) in $[7]$

One then has the following result, the proof of which is easy and was given

Proposition 7 The family $\left\{\mathcal{D}_{a}, a \in I F^{*}\right\}$ is an admissible family of dilations if and only if any $K_{a}, a \in \mathbb{I F}^{*}$ is a convolution operator

$$
K_{a} \cdot f=F_{a} * f
$$

and the discrete filters $F_{a}$ satisfy the following compatibility conditions

$$
F_{a a^{\prime}}=F_{a} *\left(D_{a} \cdot F_{a^{\prime}}\right)=F_{a^{\prime}} *\left(D_{a^{\prime}} \cdot F_{a}\right)
$$

for any $a, a^{\prime} \in I F$. Such families of filters will be called admissible.

Proof: The first argument is that the $K_{a}$ operators have to be translation invariant, which force them to be convolution operators. In addition, one must 
have $\mathcal{D}_{a a^{\prime}}=\mathcal{D}_{a} \mathcal{D}_{a^{\prime}}=\mathcal{D}_{a^{\prime}} \mathcal{D}_{a}$, which directly yields the admissibility relations.

To understand such conditions, let us assume that there exist admissible families of filters, and consider for instance the case $a=a^{\prime}=2$, and use the pseudo-dilation to compute corresponding wavelet coefficients. Let then $\psi \in \ell^{2}(I F)$, and set as usual $\tilde{\psi}(n)=\psi(-n)^{*}$. The corresponding wavelet coefficients of $f \in \ell^{2}(I F)$ read

$$
T_{f}(\cdot, 4)=f *\left[\mathcal{D}_{4} \cdot \tilde{\psi}\right]=f * K_{4} *\left[D_{4} \cdot \tilde{\psi}\right]=f * K_{2} *\left(D_{2} \cdot K_{2}\right) *\left[D_{4} \cdot \tilde{\psi}\right]
$$

This basically amounts to say the following:

- The computation of wavelet coefficients at scale 2 can be obtained by a filtering (with $D_{2} \cdot \tilde{\psi}$ ) of a "smoothed" copy of $f$, itself obtained by a smoothing with the filter $F_{2}$.

- The computation of wavelet coefficients at scale 4 can be obtained by a filtering (with $D_{4} \cdot \tilde{\psi}$ ) of a "smoothed" copy of the previous smoothing of $f$, with the filter $D_{2} \cdot F_{2}$.

- In any case, the number of multiplications is the same, since one always uses copies of the filters scaled with the $D_{a}$ dilation operator.

Then, if one wants to compute discrete wavelet transform for scales that are only powers of 2 , one immediately recovers the algorithmic structure of multiresolution analysis. Indeed, the $K_{2}$ filter is identical to the usual $H=$ $\left\{h_{n}\right\}$ filter, and the samples of the wavelet $\tilde{\psi}(n)$ play the role of the $G=\left\{g_{n}\right\}$ high-pass filter.

From the point of view of the approach developed here, the next question is that of the existence of admissible families of filters. The existence is a well-known result in the case where one only considers scales that are powers of some integral number, but not in the case of general sequences of scales. It turns out that the situation is much simpler if there exists a global scaling function, i.e. a function $\phi(x)$ such that in the Fourier space:

$$
\hat{\phi}(q \xi)=\hat{F}_{q}(\xi) \hat{\phi}(\xi)
$$

Then it is easy to see that

Proposition 8 Let $F_{q}$ be a family of discrete filters, labeled by the prime elements of $I F$, and such that there exists a function $\phi(x)$ fulfilling eq. (42) for all $q$. Let the filters $F_{a}(n), a \in \mathbb{F}^{*}$ be defined by

$$
\hat{F}_{a}(\xi)=\frac{\hat{\phi}(a \xi)}{\hat{\phi}(\xi)}
$$

Then the family $F_{a}, a \in \mathbb{I F}^{*}$ is admissible. 
Proof: Obviously, one has

$$
\hat{F}_{a a^{\prime}}(k)=\frac{\hat{\phi}\left(a a^{\prime} k\right)}{\hat{\phi}(k)}=\frac{\hat{\phi}\left(a a^{\prime} k\right)}{\hat{\phi}(a k)} \frac{\hat{\phi}(a k)}{\hat{\phi}(k)}=\hat{F}_{a^{\prime}}(a k) \hat{F}_{a}(k)
$$

which is precisely the Fourier space version of the admissibility condition.

This reduces the problem to that of finding families of filters labeled by prime numbers. It turns out that some examples of filters are easy to find out, namely those associated with spline interpolation. More precisely, taking

$$
\hat{F}_{q}(k)=\left(\frac{1-e^{\frac{2 i \pi q k}{p}}}{1-e^{\frac{2 i \pi k}{p}}}\right)^{N}
$$

we have exactly the B-spline filters, that satisfy eq.(42), in both the $\mathbb{I F}=\mathbb{Z}$ and $\mathbb{I F}=\mathbb{Z}_{p}$ cases (the $\mathbb{Z}_{p}$ case is more constrained, because of the periodicity; eq.(42) has to be fulfilled also when the product of scale factors is understood modulo $p$ ).

As an example, let us come back to the previously considered "Dirac sequence", the cyclic wavelet transform of which is now computed using a pseudo-dilation with cubic spline filters (see figure 5). The cubic spline filter "fills the holes".

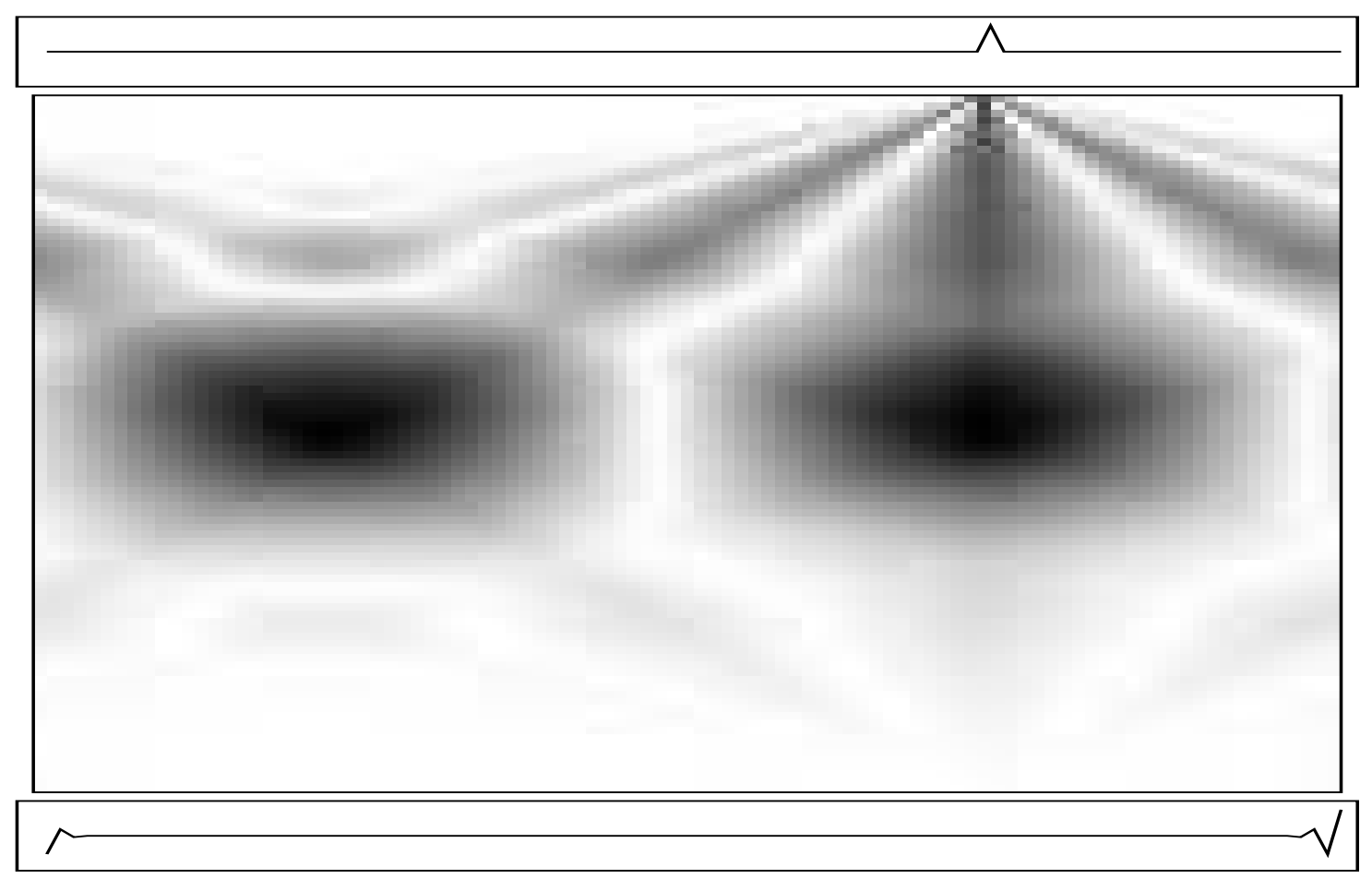

Figure 5: Cyclic wavelet transform of a "Dirac sequence", with the pseudodilation. 
As a consequence of equation (42), and since only the $F_{q}$ filters with prime scale factors $q$ are needed, one gets a pyramidal algorithm for the computation of the corresponding wavelet transform, as follows. Given a scale factor $a \in$ $I^{*}$ :

1 Decompose $a$ into prime factors

$$
a=q_{1}^{\tau_{1}} q_{2}^{\tau_{2}} \cdots q_{n}^{\tau_{n}}
$$

2 Use equation (42) to derive the decomposition of $F_{a}$ into products of scaled copies of the $F_{q_{i}}$ s.

Remark. We have only described here the algorithms for the computation of wavelet transforms $\left\langle f, \psi_{(b, a)}\right\rangle$ of some discrete signal $f \in \ell^{2}(I F)$. When $I F=\mathbb{Z}$, there does not exist a well-defined affine group acting on $\ell^{2}(\mathbb{Z})$, so that one cannot use the general approach of the first sections to get reconstruction formulas and algorithms. In the case $\mathbb{I F}=\mathbb{Z}_{p}$, the general results can be used with the $D_{a}$ dilations, yielding directly the usual reconstruction formulas. However, given a family of admissible dilations $\mathcal{D}_{a}, a \in \mathbb{Z}_{p}^{*}$, one has by construction a representation of the cyclic affine group, but it is in general not unitary. Then again the Duflo-Moore theorem cannot be used directly. It is shown in [7] how to generate a family of filters for the reconstruction algorithm.

\section{$\S$ Approximate algorithms}

We saw in the last section how the algorithmic structure of multiresolution analysis is in some sense implied by the use of dilations and the requirement of covariance with respect to dilations. The algorithms are based on the existence of a scaling function and a pair of $2 \pi$-periodic functions.

However, in many cases there does not exist such $2 \pi$-periodic filters. We shall see in this section that in some cases, it is still possible to use pyramidal algorithms, if one accepts small errors in the computation of wavelet coefficients (still keeping exact reconstruction).

\section{The one-dimensional case}

We saw in the last section how the algorithmic structure of multiresolution analysis can be derived from purely algebraic arguments. Then, as is wellknown, to get a pyramidal algorithm for the computation of wavelet coefficients, one must start from a pair $(\phi, \psi)$ of functions, with associated filters, denoted from now on $H=\left\{h_{\ell}\right\}$ and $G=\left\{g_{\ell}\right\}$ (with Fourier transforms denoted by $m_{0}(\xi)$ and $m_{1}(\xi)$ respectively), such that

$$
\hat{\phi}(2 \xi)=m_{0}(\xi) \hat{\phi}(\xi)
$$

and

$$
\hat{\psi}(2 \xi)=m_{1}(\xi) \hat{\phi}(\xi)
$$


In such cases, setting

$$
S_{j} f(n)=2^{-j} \int f(x) \phi\left(2^{-j}(x-n)\right)^{*} d x
$$

and

$$
T_{j} f(n)=2^{-j} \int f(x) \psi\left(2^{-j}(x-n)\right)^{*} d x
$$

one directly gets the following pyramidal structure for the computation of the coefficients

$$
S_{j} f(n)=\sum_{k} h_{k}^{*} S_{j-1} f\left(n-k 2^{j-1}\right)
$$

However, this is in general not the case, in the sense that although it is in general easy to associate a scaling function $\phi(x)$ to a wavelet $\psi(x)$, there is no reason to expect the quotients $\hat{\phi}(2 \xi) / \hat{\phi}(\xi)$ and $\hat{\psi}(2 \xi) / \hat{\phi}(\xi)$ to exist as $2 \pi$ periodic functions.

Nevertheless, due to the good localization of the scaling function in the Fourier space, there are good reasons to expect that such quotients (or more precisely their product with $\hat{\phi}(\xi)$ can be well-approximated by $2 \pi$-periodic functions. In other words, it may happen that there exist a pair of filters $\tilde{H}$ and $\tilde{G}$ such that the algorithm

$$
\begin{aligned}
& \tilde{T}_{j} f(n)=\sum_{k} \tilde{g}_{k}^{*} \tilde{S}_{j-1} f\left(n-2^{j-1} k\right) \\
& \tilde{S}_{j} f(n)=\sum_{k} h_{k}^{*} \tilde{S}_{j-1} f\left(n-2^{j-1} k\right)
\end{aligned}
$$

yields an accurate result. This is expressed by the following theorem

Theorem 9 Let $\psi(x)$ and $\phi(x)$ be respectively a wavelet and a scaling function such that the collection of its integer translates of $\phi(x)$ forms an unconditional basis of their closed linear span, and denote by $T_{j} f(n)$ and $S_{j} f(n)$ respectively the discrete wavelet transform and the discrete scaling function transform of $f \in L^{2}(I R)$. Then

1 There exists a unique pair of discrete filters $\tilde{H}$ and $\tilde{G}$ such that the distances $\mu_{0}=\left\|\phi(x / 2) / 2-\sum \tilde{h}_{\ell}^{*} \phi(x+\ell)\right\|_{2}$ and $\mu_{1}=\| \psi(x / 2) / 2-\sum \tilde{g}_{\ell}^{*} \phi(x+$ $\ell) \|_{2}$ are minimal.

2 These approximate filters are given by

$$
\begin{aligned}
\tilde{m}_{0}(\xi) & =\frac{\sum_{k \in \mathbb{Z}} \hat{\phi}(2(\xi+2 \pi k)) \hat{\phi}(\xi+2 \pi k)^{*}}{\sum_{k \in \mathbb{Z}}|\hat{\phi}(\xi+2 \pi k)|^{2}} \\
\tilde{m}_{1}(\xi) & =\frac{\sum_{k \in \mathbb{Z}} \hat{\psi}(2(\xi+2 \pi k)) \hat{\phi}(\xi+2 \pi k)^{*}}{\sum_{k \in \mathbb{Z}}|\hat{\phi}(\xi+2 \pi k)|^{2}}
\end{aligned}
$$


3 The following estimates hold: for any $f \in L^{2}(I R)$ :

$$
\left\|S_{j} f-\tilde{S}_{j} f\right\|_{\ell^{\infty}} \leq K_{j} \mu_{0}\|f\|_{2}
$$

and

$$
\left\|T_{j} f-\tilde{T}_{j} f\right\|_{\ell \infty} \leq K_{j}^{\prime} \mu_{1}\|f\|_{2}
$$

for some constants $K_{j}$ and $K_{j}^{\prime}$.

Proof: (sketched) The proof of existence and unicity of the $\tilde{m}_{0}$ and $\tilde{m}_{1}$ filters follows from classical arguments, and their explicit expressions are obtained by using for example the fact that $\phi(x / 2) / 2-\sum \tilde{h}_{\ell}^{*} \phi(x+\ell)$ is perpendicular (in $L^{2}$ ) to $\phi(x-k)$ for all $k \in \mathbb{Z}$ (and the same for $m_{1}$ ). The proof of the estimates are also standard (involving basically the Poisson summation formula, and the Hölder and Cauchy-Schwarz inequalities), and can be found in [16], where the asymptotic behaviour of the $K_{j}$ and $K_{j}^{\prime}$ constants is also discussed.

It is also worth noticing that since an explicit expression for the filters is available, one can easily relate the decay of the corresponding filter coefficients with the regularity of the scaling function (in the Fourier space), as follows (see [16] for a proof):

Proposition 10 If both $\psi(\xi)$ and $\hat{\phi}(\xi)$ belong to the Sobolev space $H^{m}(I R)$, then

$$
\tilde{h}_{k} \sim \tilde{g}_{k} \sim O\left(k^{-m}\right) \text { as } k \rightarrow \infty
$$

Remark. It is also interesting to notice that if the scaling function and the wavelet have been chosen in such a way that

$$
\hat{\psi}(2 \xi)=\hat{\phi}(\xi)-\hat{\phi}(2 \xi)
$$

then one has the following ("linear QMF condition")

$$
\tilde{m}_{0}+\tilde{m}_{1}=1
$$

ensuring simple reconstruction formulas:

$$
\tilde{S}_{j_{0}} f(n)=\tilde{S}_{J} f(n)+\sum_{j=J}^{j_{0}-1} \tilde{T}_{j} f(n)
$$




\section{Examples: Gaussian-type wavelets}

Let us consider for instance the case of wavelets canonically associated with Gaussian scaling functions. It is a well-known fact that in such a case, there is no multiresolution structure available, that would lead to exact pyramidal algorithms. However, the approximate filters described by theorem (??) turn out to be particularly efficient, due to the exponential localization of the scaling function in the Fourier space. Let for example the scaling function $\phi(x)$ be of the form

$$
\phi_{\alpha}(x)=K_{\alpha} e^{x^{2} / \alpha}
$$

with $K_{\alpha}$ a constant such that $\int \phi_{\alpha}(x) d x=1$, and a wavelet

$$
\psi_{\alpha}(x)=2 \phi_{\alpha}(2 x)-\phi_{\alpha}(x)
$$

one obtains a pair of approximate filters such that $\tilde{m}_{0}+\tilde{m}_{1}=1$.

The graph of $\tilde{m}_{0}(\xi)$ is shown in figure 6; filters coefficients are given in [16], and a sample Mathematica ${ }^{\mathrm{TM}}$ program for the computation of the coefficients is available [19].

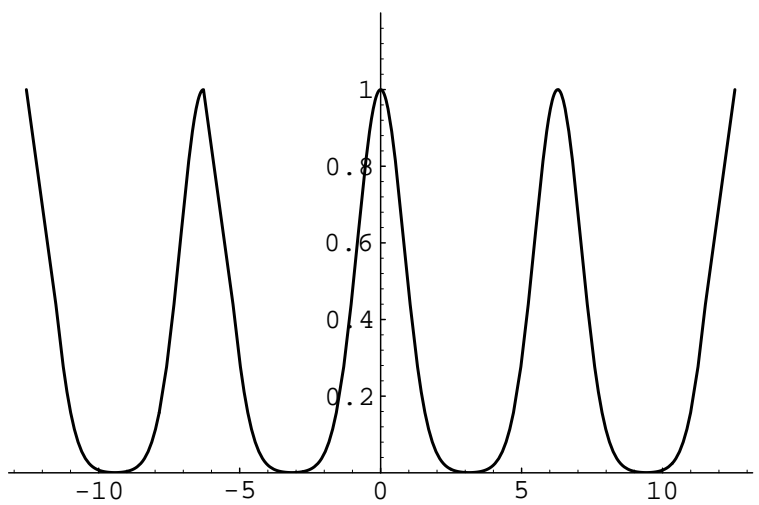

Figure 6:Approximate low-pass filter for Laplacian of Gaussian wavelet

\section{Variations on approximate filters}

In many signal analysis applications, it is desirable to also introduce redundancy with respect to the scale parameter, or to use wavelets that are more concentrated in frequency (for instance, for speech processing, one typically uses bandwidthes of one third of octave). The method developed in the previous section can also be adapted to such cases.

Let us first consider the first case, in which one is interested in getting a wavelet transform that is close to the continuous one, i.e. in which one wants to keep redundancy between scales. The simplest way to proceed in such a case is to use the previous formulas (which are all scale-covariant), and to run the algorithm starting from scaled copies of the scaling function.

More precisely, let us consider the case of a wavelet transform, with respect to wavelets of bandwidth approximatively one octave, and with $N$ different scales (in geometric progression) per octave. Set then $a_{\lambda}=a_{j, \mu}=2^{\lambda / N}$, where 
$\lambda=N j+\mu$. If $g(x)$ is an infinitesimal wavelet, normalized so that $c_{g}=1$ for simplicity, set

$$
\phi_{\mu}(x)=\int_{2^{\mu}}^{\infty} \frac{1}{a} g\left(\frac{x}{a}\right) \frac{d a}{a}
$$

and

$$
\psi_{\mu}(x)=\int_{2^{\mu-1}}^{2^{\mu}} \frac{1}{a} g\left(\frac{x}{a}\right) \frac{d a}{a}=2 \phi_{\mu}(2 x)-\phi_{\mu}(x)
$$

Then the same procedure as before will produce a family of low and high-pass filters, given by:

$$
\begin{aligned}
& \tilde{m}_{0}^{\mu}(\xi)=\frac{\sum_{k \in \mathbb{Z}} \hat{\phi}_{\mu}(2(\xi+2 \pi k)) \hat{\phi}_{\mu}(\xi+2 \pi k)^{*}}{\sum_{k \in \mathbb{Z}}\left|\hat{\phi}_{\mu}(\xi+2 \pi k)\right|^{2}} \\
& \tilde{m}_{1}^{\mu}(\xi)=\frac{\sum_{k \in \mathbb{Z}} \hat{\psi}_{\mu}(2(\xi+2 \pi k)) \hat{\phi}_{\mu}(\xi+2 \pi k)^{*}}{\sum_{k \in \mathbb{Z}}\left|\hat{\phi}_{\mu}(\xi+2 \pi k)\right|^{2}}
\end{aligned}
$$

Another possibility is to build a wavelet transform in which all sub-bands (i.e. all scales) are independent. Then it is sufficient to work with only one scaling function, and to introduce the following family of wavelets

$$
w_{\mu}=\int_{2^{(\mu-1) / N}}^{2^{\mu / N}} \frac{1}{a} g\left(\frac{x}{a}\right) \frac{d a}{a}
$$

One is then led to the following family of high-pass filters:

$$
\tilde{m}_{\mu}(\xi)=\frac{\sum_{k \in \mathbb{Z}} \hat{w}_{\mu}(2(\xi+2 \pi k)) \hat{\phi}(\xi+2 \pi k)^{*}}{\sum_{k \in \mathbb{Z}}|\hat{\phi}(\xi+2 \pi k)|^{2}}
$$

and in such a case, one has the following property:

$$
\tilde{m}_{0}+\sum \tilde{m}_{\mu}=1
$$

yielding the reconstruction formulas:

$$
S_{j_{0}} f=S_{J} f+\sum_{\mu} \sum_{j=J}^{j_{0}-1} T_{j}^{\mu} f
$$

where one has set

$$
T_{j}^{\mu} f(k)=\left\langle f, 2^{-j} w_{\mu}\left(2^{-j}(\cdot-k)\right\rangle\right.
$$

Of course, both schemes can be mixed together, for instance to get the scaleredundant third of octaves wavelet representations, that seem to be useful for speech processing. 


\section{Two-dimensional case}

The method described in the previous section yields approximate filters in cases where there does not exist "true" $2 \pi$-periodic filters for a given pair $(\phi, \psi)$. However, this situation can be generalized to the two-dimensional case, where it is for example not known how to generate filters for radial scaling functions, or to introduce angular localization in the Fourier space.

Let us for example consider the problem of constructing a pyramidal algorithm for 2-dimensional wavelets simply generated by integral translations and dilations by 2 of a unique mother wavelet. It is well-known that in such a case there does not exist any possible wavelet basis. In such cases, the same discussion as before can be developed (i.e. roughly speaking computing the orthogonal projection of $\phi(x / 2, y / 2)$ and $\psi(x / 2, y / 2)$ on the closed linear span of the functions $\phi(x-k, y-\ell))$, leading to approximate filters of the form:

$$
\begin{aligned}
& \tilde{m}_{0}(\xi, \zeta)=\frac{\sum_{k, \ell \in \mathbb{Z}} \hat{\phi}(2(\xi+2 \pi k), 2(\zeta+2 \pi \ell)) \hat{\phi}(\xi+2 \pi k, \zeta+2 \pi \ell)^{*}}{\sum_{k, \ell \in \mathbb{Z}}|\hat{\phi}(\xi+2 \pi k, \zeta+2 \pi \ell)|^{2}} \\
& \tilde{m}_{1}(\xi, \zeta)=\frac{\sum_{k, \ell \in \mathbb{Z}} \hat{\psi}(2(\xi+2 \pi k), 2(\zeta+2 \pi \ell)) \hat{\phi}(\xi+2 \pi k, \zeta+2 \pi \ell)^{*}}{\sum_{k, \ell \in \mathbb{Z}}|\hat{\phi}(\xi+2 \pi k, \zeta+2 \pi \ell)|^{2}}
\end{aligned}
$$

If one is for example interested in cases for which

$$
\hat{\psi}(2 \xi, 2 \zeta)=\hat{\phi}(\xi, \zeta)-\hat{\phi}(2 \xi, 2 \zeta)
$$

one gets "decomposition-reconstruction" algorithms of the usual form

$$
\tilde{S}_{j} f(m, n)=\sum_{k, \ell} \tilde{h}_{k, \ell} \tilde{S}_{j-1}\left(m-k 2^{j-1}, n-\ell 2^{j-1}\right)
$$

(and the same for the wavelet coefficients), and

$$
\tilde{S}_{j_{0}} f(m, n)=\tilde{S}_{J} f(m, n)+\sum \tilde{T}_{j} f(m, n)
$$

Figures 7 and 8 represent respectively the low- and high-pass filters for the two-dimensional Laplacian of Gaussian wavelets.

The problem of constructing discrete wavelet-like decompositions with angular selectivity in their frequency localization can be addressed in a similar way. For example, starting with a family of wavelets $\psi^{\lambda}(x)$ such that

$$
\sum_{\lambda} \hat{\psi}^{\lambda}(2 \xi, 2 \zeta)=\hat{\phi}(\xi, \zeta)-\hat{\phi}(2 \xi, 2 \zeta)
$$

one ends up with a family of filters $\tilde{m}_{0}(\xi, \zeta)$ (whose expression is the same as before) and $\tilde{m}_{1}^{\lambda}$, simply given by

$$
\tilde{m}_{1}^{\lambda}(\xi, \zeta)=\frac{\sum_{k, \ell \in \mathbb{Z}} \hat{\psi}^{\lambda}(2(\xi+2 \pi k), 2(\zeta+2 \pi \ell)) \hat{\phi}(\xi+2 \pi k, \zeta+2 \pi \ell)^{*}}{\sum_{k, \ell \in \mathbb{Z}}|\hat{\phi}(\xi+2 \pi k, \zeta+2 \pi \ell)|^{2}}
$$




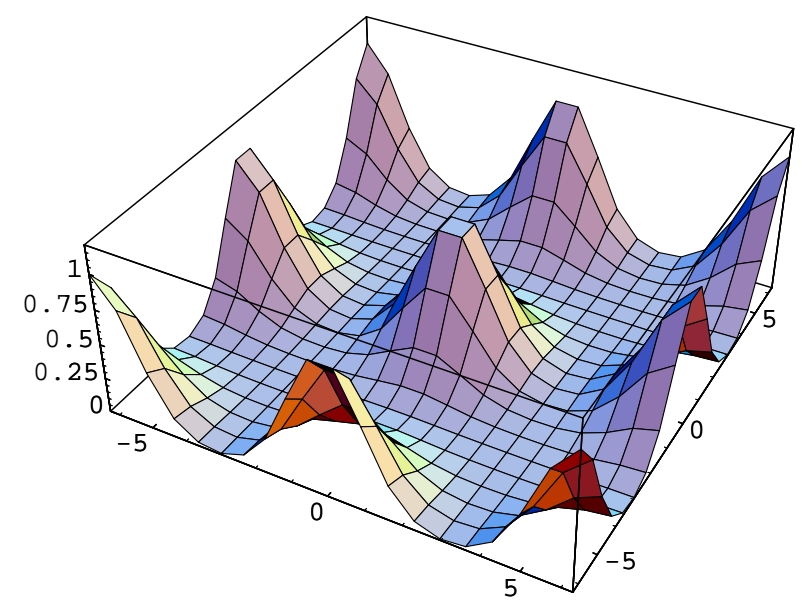

Figure 7:Approximate low-pass filter for 2D Laplacian of Gaussian wavelet

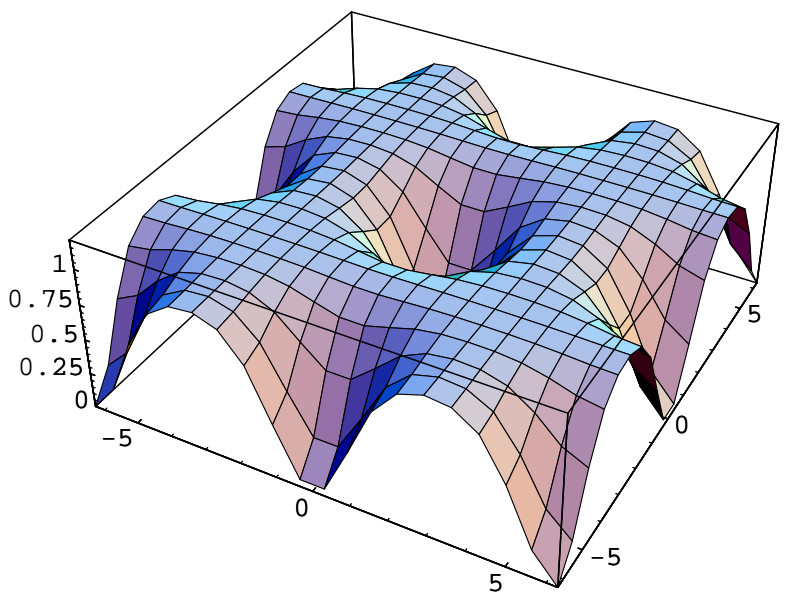

Figure 8: Approximate high-pass filter for 2D Laplacian of Gaussian (radial) wavelet

Remark. It is important to point out that the methodology developed in this section only aims to produce pyramidal algorithms with exact reconstruction, with the corresponding discrete filters. Since the construction actually starts from a wavelet and a scaling function, it is also natural to expect the coefficients produced by the algorithm to be somewhat close to the corresponding wavelet ans scaling function coefficients. This is quite natural in the 1D case, as we saw in the last section. In the $2 \mathrm{D}$ radial case, this needs not be the case, the square geometry imposed by the square lattice the algorithms are built on being not really adapted to the radial assumptions. Otherwise stated, the $\mu_{0}$ and $\mu_{1}$ coefficients may be larger than in the $1 \mathrm{D}$ case. Along the same ideas, it sounds natural to expect a better situation if one works with hexagonal lattice instead of the square one (the formulas we gave here are easily modified accordingly). Up to now we have actually no proof of this.

Acknowledgments. Many of the results described in this paper have been obtained in collaboration with M. Duval-Destin, K. Flornes, C. Gonnet, A. Grossmann, C. Kalisa, M. Holschneider, M.A. Muschietti. I would like to take 
the opportunity to thank them for the pleasure of collaboration. I would also like to thank J.P. Antoine, G. Beylkin, Y. Meyer and R. Murenzi for fruitful discussions. This work was supported in part by the late "GDR Ondelettes", CNRS.

\section{References}

1. Ali, S.T., Antoine, J.P., Gazeau, J.P., Square-integrability of group representations on homogeneous spaces I, Reproducing triples and frames, Ann. Inst. H. Poincaré 55 (1991) 829-856; II, Generalized square-integrability and equivalent families of coherent states, Ann. Inst. H. Poincaré 55 (1991) 860-890.

2. Antoine, J.P., Carrette, B., Murenzi, R., Piette, B., Image analysis with 2-dimensional wavelet transform, Signal Processing 31 (1993) 241-272.

3. Bernier, D. and Taylor, K.F., Wavelets from square-integrable representations, Preprint, Dept of Mathematics and Statistics, University of Saskatchewan, Saskatoon, Sask. S7N 0W0, Canada.

4. Carey, A.L., Square-integrable representations of non-unimodular groups, Bull. Austr. Math. Soc. 15 (1976) 1.

5. De Bièvre, S., Coherent states over symplectic homogeneous spaces, J. Math. Phys. 30 (1989) 1401-1407.

6. Duflo, M., Moore, C.C., On the regular representation of a nonunimodular locally compact group, J. Funct. An. 21 (1976) 209-243.

7. Flornes, K., Grossmann, A., Holschneider, M. and Torrésani, B., Wavelets on finite fields, Preprint, to appear at Appl. and Comp. Harmonic Anal.

8. Holschneider, M., Kronland-Martinet, R., Morlet, J., Tchamitchian, Ph., A real-time algorithm for signal analysis with the help of wavelet transform, in Wavelets, Time-Frequency methods and Phase Space, J.M. Combes, A. Grossmann, Ph. Tchamitchian Eds., Marseille (1987), IPTI, Springer, p. 286-297.

9. Gonnet, C., and Torrésani, B., Local frequency analysis with the help of two-dimensional wavelet transform, Preprint, To appear in Signal Processing (1994).

10. Grossmann, A., Morlet, J., and Paul, T., Transforms associated with square integrable group representations I, J. Math. Phys. 27 (1985),2473. II, Ann. Inst. H. Poincaré, 45 (1986) 293.

11. Kalisa, C. and Torrésani, B., N-dimensional affine Weyl-Heisenberg wavelets, Preprint, to appear at Ann. Inst. H. Poincaré, Phys. Théor. (1994).

12. Kirillov, A., Elements of representation theory, Moscow.

13. Mallat, S., A theory for multiresolution signal decomposition: the wavelet representation, IEEE Pattern Anal. and Machine Intell. 11 (7) (1989), 674-693.

14. Meyer, Y., Ondelettes et Opérateurs, in two volumes, Hermann, Paris, 1990.

15. Murenzi, R., Ondelettes multidimensionnelles et application à l'analyse d'image, PhD Thesis, Louvain-la-Neuve, Belgium. 
16. Muschietti, M.A and Torrésani, B., Pyramidal algorithms for LittlewoodPaley decompositions, Preprint, to appear at SIAM J. Math. An (1994).

17. Torrésani, B., Time-Frequency Representations: wavelet packets and optimal decomposition, Ann. Inst. H. Poincaré, Phys. Théor. 56 (1992), 1.

18. Torrésani, B., Phase-space decompositions; local Fourier analysis on spheres, Preprint, CPT Marseille, submitted to Appl. and Comp. Harmonic Anal..

19. Torrésani, B., Approximate filters for LOG wavelets, Mathematica ${ }^{\mathrm{TM}}$ program, available by anonymous ftp at cpt.univ-mrs.fr (internet number 139.124.7.5), directory /pub/preprints/94/wavelets , file LOG.ma.

\section{Bruno TORRESANI}

CPT, CNRS-Luminy, Case 907, 13288 Marseille Cedex 09, France. torresan@marcptnx1.univ-mrs.fr 